\title{
Non-histone protein HMGB1 inhibits the repair of damaged DNA by cisplatin in NIH-3T3 murine fibroblasts
}

\author{
Shazie Yusein-Myashkova, Iva Ugrinova \& Evdokia Pasheva ${ }^{*}$ \\ Institute of Molecular Biology, Roumen Tsanev, Bulgarian Academy of Sciences, Sofia 1113, Bulgaria
}

\begin{abstract}
The nuclear non-histone protein high mobility group box (HMGB) 1 is known to having an inhibitory effect on the repair of DNA damaged by the antitumor drug cisplatin in vitro. To investigate the role of HMGB1 in living cells, we studied the DNA repair of cisplatin damages in mouse fibroblast cell line, NIH-3T3. We evaluated the effect of the post-synthetic acetylation and C-terminal domain of the protein by overexpression of the parental and mutant GFP fused forms of HMGB1. The results revealed that HMGB1 had also an inhibitory effect on the repair of cisplatin damaged DNA in vivo. The silencing of HMGB1 in NIH-3T3 cells increased the cellular DNA repair potential. The increased levels of repair synthesis could be "rescued" and returned to less than normal levels if the knockdown cells were transfected with plasmids encoding HMGB1 and HMGB1 K2A. In this case, the truncated form of HMGB1 also exhibited a slight inhibitory effect. [BMB Reports 2016; 49(2): 99-104]
\end{abstract}

\section{INTRODUCTION}

The high mobility group box (HMGB) 1 is an abundant, evolutionarily conserved non-histone protein, which contains two DNA-binding domains (HMG-boxes A and B) and a long acidic C-terminal tail (1). HMGB1 implicated in DNA repair pathways (reviewed in 2). The protein binds lesions induced by UV light (3) and by the chemotherapeutic agent cisplatin (4-6). Such damages are removed by the nucleotide excision repair (NER). HMGB1 not only recognizes and binds UV and cisplatin lesions, but also interacts functionally with several protein components involved in $\operatorname{NER}(7,8)$. In vitro studies have shown that HMGB1 inhibits the repair of cis-platinated DNA $(6,9,10)$. One possible mechanism might be the protection of

*Corresponding author. Tel: +3592-9792668; Fax: +3592-8723507; E-mail: eva@bio21.bas.bg

http://dx.doi.org/10.5483/BMBRep.2016.49.2.238

Received 30 October 2013, Revised 20 November 2013, Accepted 11 November 2013

Keywords: Acetylation, Cisplatin, HMGB1, NER, Tail-less HMGB1 protein cisplatin adducts from repair by the DNA-HMGB1 complexes. The "shielding" effect prevents the access of NER factors to the cisplatin lesions. In agreement with this, is the observation that binding of HMGB1 to the DNA defect inhibits the interaction of NER protein RPA to the target lesion (11). Another hypothesis suggested that the effect of the C-terminus was accomplished through protein-protein interaction(s) (6). Other HMG box containing proteins were also reported to recognize and inhibit the removal of intra-strand DNA cross-links when added to in vitro repair assay $(9,12,13)$. On the other hand, reduced histone acetylation after DNA damage in HMGB1-deficient cells indicated a role of HMGB1 in DNA damage-induced chromatin remodelling (14). It is possible that this influence is p53-dependent as HMGB1 is found to interact with the tumour suppressor protein thus facilitating its binding to DNA (15-17). p53 up-regulates Gadd45 and binds the histone acetyltransferase p300, events required to stimulate chromatin relaxation, which in turn facilitates access of global genome nucleotide excision repair (GG-NER) recognition proteins to damaged heterochromatin within the overall genome $(18,19)$. HMGB1 protein was linked to the action of other anti-cancer drugs as doxorubicin and methotrexate by the mechanism of autophagy (20).

Post-translational acetylation of HMGB1 at Lys 2 affects its chromatin interaction and binding affinity to DNA $(6,21,22)$. It was also found that Lysine 2 physically interacted with the acidic tail of the protein (23) and its acetylation might disrupt this interaction. The C-terminal domain has a modulatory function as it intra-molecularly associates with HMG-boxes and regulates their availability for DNA binding (24-26). The acidic C-tail of HMGB1 also participates in protein-protein interactions $(23,27-31)$ and is necessary for nucleosome sliding (32). It is possible that the acetylation of Lys 2 contributes for more efficient DNA binding of the HMG boxes to the damaged DNA and/or release the acidic C-tail responsible for protein-protein interactions. It is important to understand the contribution of Lys 2 acetylation and the acidic tail for the effect of HMGB1 protein on DNA repair for better understanding how HMGB1 modulates this nuclear process. The literature data suggested that HMGB1 could influence the DNA repair in the living cells through different mechanisms. However, few studies investigated the effect of the protein at the cellular level. To clarify further what is the role of HMGB1 and its modifications

ISSN: 1976-670X (electronic edition)

Copyright (C) 2016 by the The Korean Society for Biochemistry and Molecular Biology

(c) This is an open-access article distributed under the terms of the Creative Commons Attribution Non-Commercial License (http://creativecommons.org/licenses/by-nc/4.0) which permits unrestricted non-commercial use, distribution, and reproduction in any medium, provided the original work is properly cited. 
A

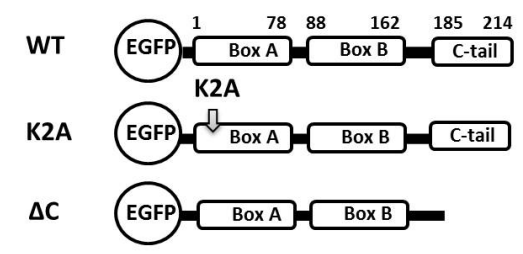

B

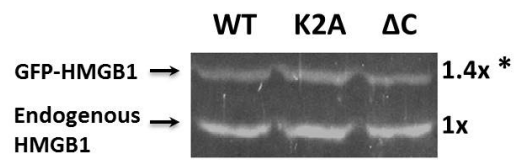

* 25-30\% transfection levels
C
GFP

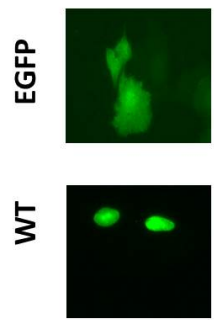

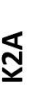

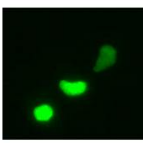

y

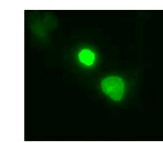

DAPI
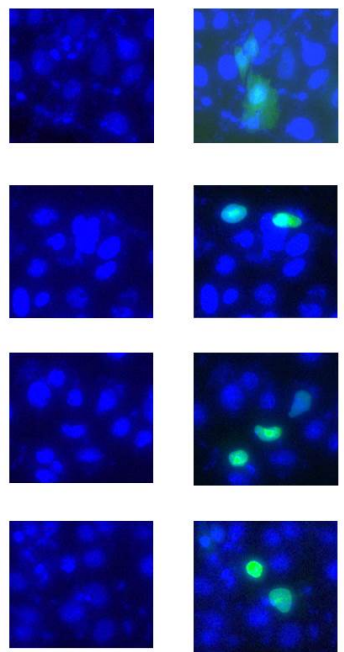

Fig. 1. Expression of GFP-HMGB1 constructs and their cellular localization. (A) WT: wild type full length HMGB1, K2A: HMGB1 mutated at the acetylation site $\mathrm{K} 2$ and $\triangle \mathrm{C}$ : HMGB1 lacking C-terminal domain. (B) western blot analysis of GFP-HMGB1 forms transfected in NIH-3T3 cells. (C) microscopic data for GFP and GFPHMGB1 cellular localization in NIH$3 \mathrm{~T} 3$ cells. for the DNA repair of mammalian cells, we studied the repair of cisplatin damaged DNA in mouse fibroblast cell line $\mathrm{NIH}-3 \mathrm{~T} 3$ upon overexpression of the wild protein, mutant form K2A (mutated at site of acetylation Lys 2) and the tailless form. We used a host-cell reactivation assay (HCR) in order to monitor the repair of DNA in normal and HMGB1 knockdown mouse fibroblast cell lines.

\section{RESULTS}

Over-expression of HMGB1 in NIH-3T3 cells inhibits the repair of damaged DNA with the antitumor drug cisplatin To evaluate the effect of HMGB1 protein and the role of acetylation and the C-terminal domain on the DNA repair, we created three plasmid constructs: WT-encoding wild type full length protein, K2A-mutated in Lys2 acetylation site and $\Delta \mathrm{C}$ : HMGB1 lacking its C-terminal domai. All were cloned in EGFP-C1 vector (Fig. 1A). The transfection level of the three GFP constructs was comparable (25-30\%) as well as the amount of the synthesized fusion proteins demonstrated by western blot analysis (Fig. 1B). The relative endogenous HMGB1 level and the three exogenous GFP fusion proteins' levels were quantified using Odyssey software and normalized to the number of transfected cells. In all cases, the amount of the exogenous GFP fused proteins was approximately 1.4 times greater than the cellular HMGB1. All fussed proteins showed nuclear localization (Fig. 1C). The three forms were expressed in $\mathrm{NIH}-3 \mathrm{~T} 3$ cell line and their effects on the cellular repair capacity of cis-platinated pDsRedEx2C1 plasmid encoding red fluorescent protein were analysed. Cells expressing GFP alone were used as a control to eliminate the effect, if any, of the green fluorescent protein. The percentage of the successful transfection of both EGFP-C1 and

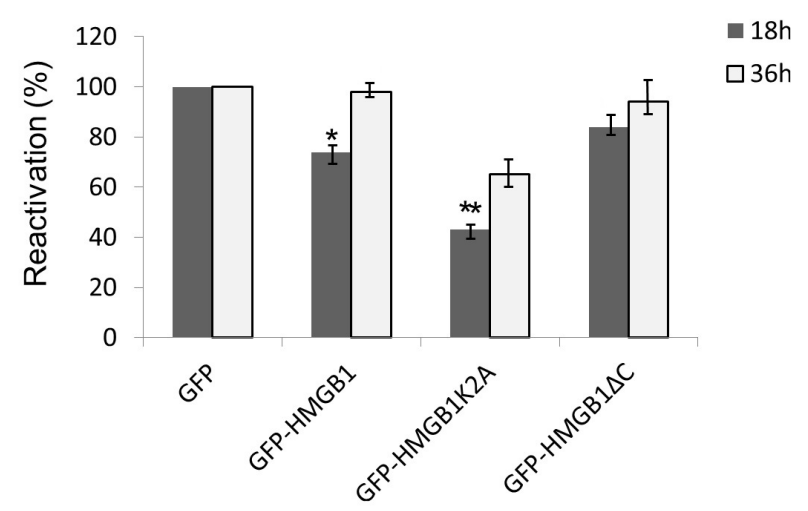

Fig. 2. Host cell reactivation assay in NIH-3T3 line in the presence of GFP-HMGB1 forms. The cells were transfected with pDsRedExC1 damaged with cisplatin in molar ratio 1:50 and GFP-HMGB1. The number of red fluorescent cells with damaged plasmid was measured $16 \mathrm{~h}$ and $32 \mathrm{~h}$ after transfection and compared to the number of DsRed positive cells with undamaged plasmid for the estimation of reactivation. The NIH-3T3 cells expressing GFP alone were used for the normalization of data. Asterisks indicate differences between the reactivation in HMGB1 forms expressing cells and the control with $* \mathrm{P}<0.05$ or $* * \mathrm{P}<0.01$. Student t-test for comparisons between groups was used. The results are from two independent experiments.

pDsRedEx2C1 vectors was comparable (data not shown). Only double positive cells (red and green) were calculated for the host reactivation (HR) assay. All data were normalized to the reactivation in the control cells. The results presented in Fig. 2 showed that the full length HMGB1 and the mutated HMGB1 K2A had a well defined inhibitory effect on the DNA repair 
whereas the C-terminal lacking form revealed negligible decrease. To optimize the conditions for the HR assay, we did experiments with different levels (1:50 and 1:70) of cis-platinated DsRed plasmid (Fig. S1, A). The repair potential of the cells expectedly depended on the damaged DNA level. The HR inhibition pattern of the three forms of GFP-HMGB1 fusion proteins on the repair of cis-platinated DsRed plasmids (1:50, 1:70) was the same suggesting that the effects of the HMGB1 proteins were independent of damage level (Fig.1S, B).

\section{Down-regulation of HMGB1 in NIH-3T3 cells enhances the} repair of damaged DNA by cisplatin

We generated $\mathrm{NIH}-3 \mathrm{~T} 3$ cells with significant down-regulation of HMGB1 using shRNA construct to diminish the effect of the endogenous HMGB1 protein and estimate more precisely the in vivo effect of HMGB1, its mutated, and truncated form. After several transfections and selection with puromycin, we
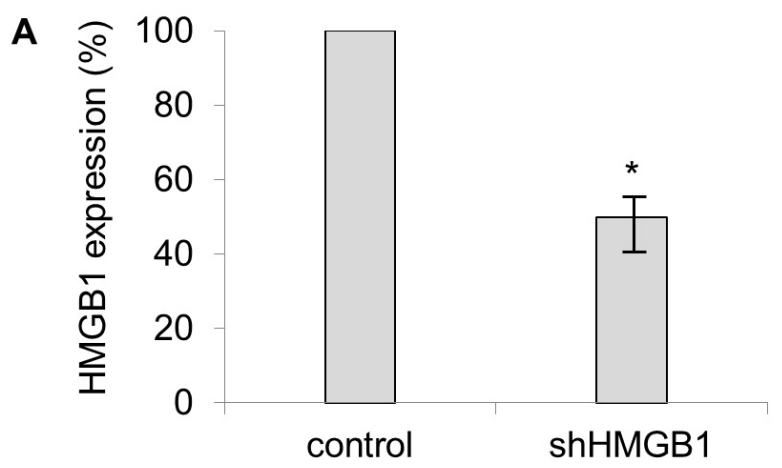

B

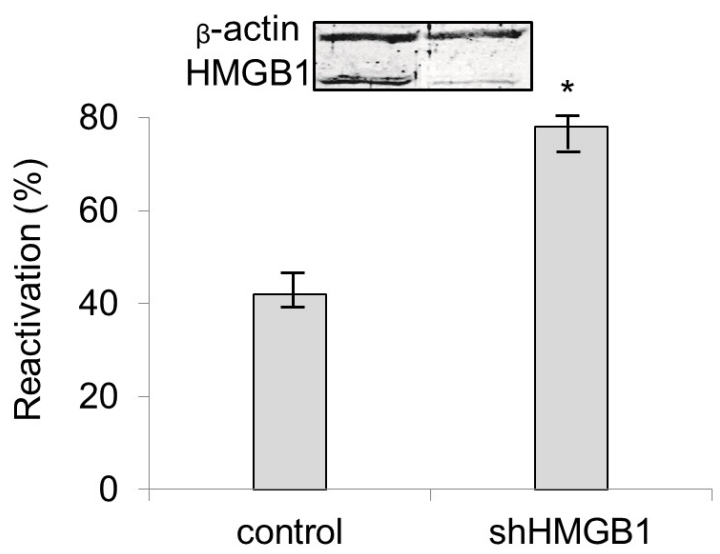

Fig. 3. Down-regulation of HMGB1 enhances the repair of damaged DNA by cisplatin. (A) Western blot analysis showed a decrease in the intracellular levels of HMGB1 with $60 \%$ in stable knock-down cell line in comparison to the control cells. The relative HMGB1 levels were quantified using Odyssey software and were normalized to $\beta$-actin expression. (B) Host cell reactivation assay of cis-platinated pDsRedExC1 in normal and knock-downed cells. ${ }^{*} \mathrm{P}<0.05$ generated a cell line with $60 \%$ decreased HMGB1 protein levels analysed by western blot (Fig. 3A). The DNA repair capacity of control 3T3 cells and HMGB1 knocked-down cells was compared. HR assay showed that down-regulation of HMGB1 enhanced the repair of damaged DNA and the effect was well defined $16 \mathrm{~h}$ after transfection (Fig. 3B).

We overexpressed the three GFP HMGB1 forms in knockeddown NIH-3T3 cells and analysed their effect on the repair of cis-platinated DNA 16 hours after transfection when the increase of the repair potential of HMGB1 knocked-down 3T3 cells was more prominent. The addition of exogenous GFP HMGB1 inhibited the repair synthesis even less compared to the levels in control cells (Fig. 4). However, K2A mutant form, which inhibitory effect was the most notable in the non-silenced cells (Fig. 2), showed the same tendency in the silenced cells too (Fig. 4). We registered interesting results after overexpression of HMGB1 lacking C-terminal domain. Whereas in non-silenced cells, this form did not have an effect on DNA repair capacity, in silenced cells, it showed slight inhibitory effect (Fig. 4). It is worth mentioning that in the knock-downed NIH-3T3 cells, we observed $5-10 \%$ of dead cells measured by staining with trypan blue - the same value as in the "normal" cells after transfection which indicated that the viability of the cells was not affected.

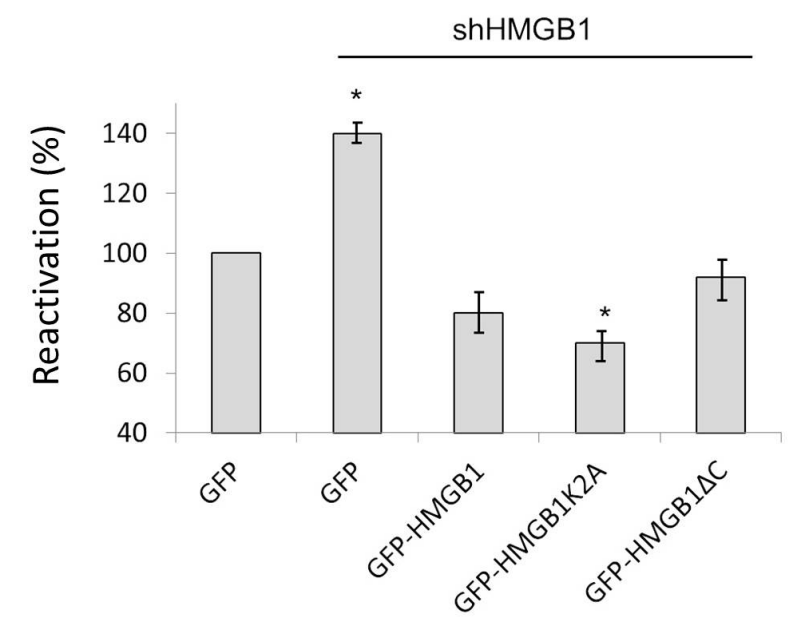

Fig. 4. Host cell reactivation assay in NIH-3T3 HMGB1 knock-down line in the presence of GFP-HMGB1 forms. The cells were transfected with cis-platinated pDsRedExC1 (1:50) and HMGB1 forms fused with EGFP (WT, mutated K2A and lacking C-terminal domain- $\Delta C$ ). The reactivation in control (3T3 cells, expressing GFP alone) is used for the normalization of data (given in percentage with SEM). Asterisks indicate differences between the reactivation in HMGB1 forms expressing cells and the control with $* P<0.05$. Student t-test for comparisons between groups was used. 


\section{DISCUSSION}

HMGB1 protein specifically recognizes and binds to UV lesions (3) and DNA damaged by the anticancer drug cisplatin $(4-6,21)$, and the affinity of HMGB1 to the lesions is modulated by acetylation (21) and the acidic C-tail (6). Several published data $(7,8)$ demonstrated that HMGB1 protein is involved in NER pathway. The protein inhibits the repair of cis-platinated DNA in vitro $(6,9)$. One possible mechanism for the explanation of this finding is the protection of cisplatin lesions from the repair machinery. However the inhibitory effect of HMGB1 does not correlate with the binding affinity of the protein to the lesion, but is modulated by its C-terminal domain (6). Our results demonstrated that HMGB1 also inhibited the DNA repair in vivo in NIH-3T3 cells. Furthermore, we confirmed the previous findings in cell free extracts (6) that HMGB1 lacking the acidic tail did not inhibit the repair in presence of endogenous HMGB1. The mutated form (K2A) that cannot undergo acetylation, had higher capacity to inhibit the repair than the wild type form in non-silenced cells. Our previously stated hypothesis was (6) that the $C$ terminus was responsible for the inhibitory effect by protein/protein interactions. In case of acetylation or mutation of the lysine at position 2, the C tail becomes "free" and functional as its physical contact with Lys2 is disrupted (23).

One experimental approach to clarify the role of HMGB1 protein and its $\mathrm{C}$ terminus in vivo is to knock-down HMGB1 in $\mathrm{NIH}-3 \mathrm{~T} 3$ cells in order to eliminate the impact of the endogenous protein. We succeeded in diminishing the cellular HMGB1 level up to $60 \%$. The first important result that we observed, was the stimulation of the DNA repair capacity of the cell line. That finding was an indirect indication that the protein exerted inhibitory effect on the repair process of cis-platinated DNA. When the silenced cells were transfected with the three forms: GFP-HMGB1, GFP-HMGB1 (K2) and GFP-HMGB1 $\Delta$ C, the repair synthesis diminished under the level in the normal NIH-3T3 cells for the first two forms. The less pronounced inhibition of the mutated HMGB1 in the knock-downed cells compared to the normal ones might be explained by a cumulative effect of the exogenous mutated and endogenous HMGB1 protein in the normal $\mathrm{NIH}-3 \mathrm{~T} 3$ cells. An interesting observation was that the repair potential of the silenced NIH-3T3 cells was slightly decreased in the presence of GFP-HMGB1 $\Delta \mathrm{C}$. One possible explanation might be that when the concurrence of the endogenous HMGB1 was overcome, the inhibitory effect of the truncated protein on the remodelling dominated (32) as plasmid DNA adopts a nucleosomal organization following transient transfections (33).

We may speculate that the inhibitory effect of HMGB1 in the DNA damage response is accomplished through capturing factors from the repair machinery away from the damages or suppressing their function. The binding partners of HMGB1 include numerous proteins including NER proteins RPA, XPA and XPC $(7,8)$. The inhibitory effect of HMGB1 in our study was detectable only in the early hours after transfections of normal cells and was compensated at the $36^{\text {th }}$ hour. One possible explanation is the activation of the transcription of the repair genes and their accumulation in the cell at the late hours. The biosynthesis of DNA damage sensors directly dictates the capacity of DNA lesion removal by NER. A critical threshold regulatory function has been ascribed to $\mathrm{p} 53$, which governs transcription and thus maintains basal-level expression of rate-limiting human DDB2 and XPC damage sensors under normal growth conditions (34). p53-deficient cells contain reduced levels of DDB2 and XPC, and display diminished GG-NER activity (34-36). So it is possible for HMGB1 to inhibit the DNA repair by binding the repair proteins when they are at basal levels but not at up-regulated levels. Studies revealed that more than 24 hours are needed for the maximum accumulation of proteins whose transcription is induced by p53 after DNA damage (34). Our findings suggest that when estimating the involvement of HMGB1 protein in the DNA repair response and the role of the $C$ terminus in the living cells, we should consider it as a complex phenomenon affecting numerous nuclear processes as repair, remodelling, transcription etc.

\section{MATERIALS AND METHODS}

\section{Generation of stable HMGB1 knockdown cell lines}

Mouse fibroblast (NIH-3T3) cells were maintained in DMEM (Invitrogen) supplemented with 10\% fetal bovine serum (Invitrogen) and Penicillin/Streptomycin (Invitrogen) in $5 \% \mathrm{CO}_{2}$ at $37^{\circ} \mathrm{C}$. A short hairpin RNA construct was made in pBabeH1 vector (pBabeH1-HMGB1) using the sequence: 59-UGCUGC CUACAGAGCUAAA-39, targeting mouse HMGB1 was kindly provided by Dr. E. Abraham (37). Stable NIH3T3 cell lines with down-regulation of HMGB1 were generated by several transfections with pBabeH1-HMGB1 plasmid DNA using Lipofectamine 2,000 reagent (Invitrogen) followed by selection with puromycin $(2 \mathrm{mg} / \mathrm{ml})$.

\section{Plasmid treatment with cisplatin}

Plasmid pDsRedEx2C1 (Clontech) containing reporter gene DsRed-Express2 for the red fluorescent protein was propagated in E. coli strain DH5 $\alpha$. Plasmid DNA in TE buffer was incubated overnight on room temperature with cisplatin (Sigma) in different molar ratios. As previously described in (6) cisplatin treated plasmid DNA in molar ratio 50:1 contained 20 atoms platinum bound per 1,000 bp.

\section{Plasmid constructions}

Full length, mutant $(\mathrm{K} 2 \mathrm{~A})$ and truncated $(\Delta \mathrm{C})$ forms of HMGB1 were cloned by PCR-based sub-cloning using primers containing BgIII and EcoRI restriction sites for full length (214 aa), mutant K2A and $\Delta C$ truncated (184 aa) in pET28a (Novagen) plasmid as a template. The PCR was carried out using Vent DNA polymerase (NEB) at $95^{\circ} \mathrm{C} / 5 \mathrm{~min} ; 30$ cycles $\left(95^{\circ} \mathrm{C} / 30 \mathrm{~s}\right.$, 
$\left.57^{\circ} \mathrm{C} / 40 \mathrm{~s}, 72^{\circ} \mathrm{C} / 60 \mathrm{~s}\right) ; 72^{\circ} \mathrm{C} / 5 \mathrm{~min}$. The amplicons were inserted between Bglll and EcoRI sites of pEGFP-C1 (Clontech), ligated, propagated in $\mathrm{E}$. coli strain $\mathrm{DH} 5 \alpha$ and purified.

\section{Host cell reactivation (HCR) assay}

Fifty percent confluent NIH-3T3 cells in 96 well plates were transfected with $0.4 \mu \mathrm{l}$ LipofectaminTM 2000 (Invitrogen) and $0.15 \mu \mathrm{g}$ damaged and undamaged plasmid pDsRedEx2C1 (Clontech) plasmid DNA encoding red fluorescent protein. Cells were also co-transfected with equal quantity GFP plasmids. For each experiment, one well from 96 wells plate per DNA mixture was transfected containing pDsRedExC1 (damaged or undamaged) and pEGFP-C1 (GFP alone or fused with HMGB1 forms). After 18 or 36 hours, cells were imaged at magnification 10x and recorded with differential interference contrast (DIC) and filters for green and red fluorescence. Five regions per well were photographed and fluorescent and nonfluorescent cells were counted ( $\sim 1,500$ cells per experiment). The average persentage of fluorescent cells were used for calculation of the reactivation $(R)$ by the formula: $R=$ red cells with damaged plasmid/ red cells with undamaged plasmid $\times 100$.

\section{Microscopy}

Fibroblasts were seeded on coverslips placed in 24 well plates and transfected with $2 \mu$ l LipofectaminTM 2000 (Invitrogen) and $0.75 \mu \mathrm{g}$ plasmid DNA (pEGFP-C1 or pEGFPC1-HMGB1). After 24 hours, cells were washed with PBS and fixed in paraformaldehyde. Coverslips were inverted onto the drop of mounting media containing DAPI (UltraCruz ${ }^{\text {TM }}$ Mounting Medium, Santa Cruz). Cells were observed with Axiovert 200M inverted fluorescent microscope (Carl Zeiss) using AxioCam MRm CCD camera (Carl Zeiss) and Carl Zeiss filters set 38HE (excitation: BP 470/40; beamsplitter: FT 495; emission: BP 525/50) for GFP signals, set 15 (excitation: BP 549/12; beamsplitter: FT 580; emission: LP 590) for DsRed signals and set 01 (excitation: BP 365/12; beamsplitter: FT 395; emission: LP 397) for DAPI signals. Images were acquired and processed by Carl Zeiss AxioVision Rel.4.7 software.

\section{Western blot analysis}

$\mathrm{NIH}-3 \mathrm{~T} 3$ cells were harvested, lysed in Nonidet-P40 buffer and denatured in sample buffer $(50 \mathrm{mM}$ Tris $\mathrm{HCl} \mathrm{pH} 6.8 ; 2 \%$ SDS; $10 \%$ glycerol; $1 \% \beta$-mercaptoethanol; $12.5 \mathrm{mM}$ EDTA, $0.02 \%$ bromphenol blue). Proteins were run on a $12 \%$ sodium dodecyl sulfate-polyacrylamide electrophoresis gel and transferred to nitrocellulose membranes. HMGB1 quantities were detected on a protein blot using polyclonal anti-HMGB1 (Millipore $1: 1,000)$ and goat anti-rabbit IRDye800CW secondary antibody (LI-COR; 1:10,000). $\beta$-actin was detected with monoclonal $\beta$-actin (Thermoscientific $1: 2,000$ ) and goat anti-mouse IRDye 680 secondary antibody (LI-COR; 1:10,000). The bands were visualized using the Odyssey Infrared Imaging System (LI-COR). The relative HMGB1 levels were quantified using Odyssey software and were normalized to $\beta$-actin expression.

\section{ACKNOWLEDGEMENTS}

We thank Dr. E. Abraham for the shRNA construct pBabeH1HMGB1. This work was supported by a grant DTK $02 / 80$ from the Bulgarian Science Fund.

\section{REFERENCES}

1. Goodwin GH, Sanders C and Johns EW (1973) A new group of chromatin-associated proteins with a high content of acidic and basic amino acids. Eur J Biochem 38, 14-19

2. Reeves R and Adair JE (2005) Role of high mobility group (HMG) chromatin proteins in DNA repair. DNA Repair 4, 926-938

3. Pasheva EA, Pashev IG and Favre A (1998) Preferential binding of high mobility group 1 protein to UV damaged DNA. J Biol Chem 273, 24730-24736

4. Pil PM and Lippard SJ (1992) Specific binding of chromosomal protein HMG1 to DNA damaged by the anticancer drug cisplatin. Science 256, 234-237

5. Hughes EN, Engelsberg BN and Billings PC (1992) Purification of nuclear proteins that bind to cisplatin-damaged DNA. Identity with high mobility group proteins 1 and 2. J Biol Chem 267, 13520-13527

6. Mitkova E, Ugrinova I, Pashev I and Pasheva E (2005) The inhibitory effect of HMGB1 protein on the repair of cisplatin damaged DNA is accomplished through the acidic domain. Biochemistry 44, 5893-5898

7. Lange SS, Reddy MC and Vasquez KM (2009) Human HMGB1 directly facilitates interactions between nucleotide excision repair proteins on triplex-directed psoralen interstrand crosslinks. DNA Repair 8, 865-72

8. Reddy MC, Christensen J and Vasquez KM (2005) Interplay between human high mobility group protein 1 and replication protein A on psoralen-cross-linked DNA. Biochemistry $44,4188-4195$

9. Huang JC, Zamble DB, Reardon RT et al (1994) HMG-domain proteins specifically inhibit the repair of the major DNA adduct of the anticancer drug cisplatin by human excision nuclease. Proc Natl Acad Sci U S A 91, 1039410398

10. Malina J, Kasparkova J, Natile G and Brabec V (2002) Recognition of major DNA adducts of enantiomeric cisplatin analogs by HMG box proteins and nucleotide excision repair of these adducts. Chem Biol 9, 629-638

11. Patrick SM and Turchi JJ (1998) Human replication protein A preferentially binds cisplatin-damaged duplex DNA in vitro. Biochemistry 37, 8808-8815

12. Zamble DB, Mu D, Reardon JT et al (1996) Repair of cisplatin-DNA adducts by the mammalian excision nuclease. Biochemistry 35, 10004-10013

13. Trimmer EE and Essigmann JM (1998) Human testis-determining factor SRY binds to the major DNA adducts of cisplatin and a putative target sequence with comparable affinities. Biochemistry 37, 352-362

14. Lange SS, Mitchell DL and Vasquez KM (2008) High mobility group protein $B 1$ enhances DNA repair and chromatin modification after DNA damage. Proc Natl Acad Sci U 
S A 105 (30), 10320-10325

15. Jayaraman L, Moorthy NC, Murthy KG et al (1998) High mobility group protein-1 (HMG-1) is a unique activator of p53. Genes Dev 12, 462-472

16. Banerjee $S$ and Kundu TK (2003) The acidic C-terminal domain and A-box of HMGB-1 regulates p53-mediated transcription. Nucleic Acids Res 31, 3236-3247

17. Stros M, Muselikova-Polanska E, Pospisilova $S$ and Strauss F (2004) High-affinity binding of tumor-suppressor protein p53 and HMGB1 to hemicatenated DNA loops. Biochemistry 43, 7215-7225

18. Smith ML, Ford JM, Hollander MC et al (2000) p53-mediated DNA repair responses to UV radiation: studies of mouse cells lacking p53, p21, and/or gadd45 genes. Mol Cell Biol 20, 3705-3714

19. Rubbi CP and Milner J (2003) p53 is a chromatin accessibility factor for nucleotide excision repair of DNA damage. EMBO J 22, 975-986

20. Huang J, Ni J, Liu K et al (2012) HMGB1 Promotes Drug Resistance in Osteosarcoma. Cancer Res 72, 230-238

21. Ugrinova I, Pasheva EA, Armengaud J and Pashev IG (2001) In vivo acetylation of HMG1 protein enhances its binding affinity to distorted DNA structures. Biochemistry 40, 14655-14660

22. Assenberg R, Webb M, Connolly E et al (2008) A critical role in structure-specific DNA binding for the acetylatable lysine residues in HMGB1. Biochem J 411, 553-561

23. Ueda T, Chou H, Kawase $T$ et al (2004) Acidic C-tail of HMGB1 is required for its target binding to nucleosome linker DNA and transcription stimulation. Biochemistry 43, 9901-9908

24. Knapp S, Muller S, Digilio G et al (2004) The long acidic tail of high mobility group box 1 (HMGB1) protein forms an extended and flexible structure that interacts with specific residues within and between the HMG boxes. Biochemistry 43, 11992-11997

25. Watson M, Stott K, Thomas JO (2007) Mapping intramolecular interactions between domains in HMGB1 using a tail-truncation approach. J Mol Biol 374, 1286-1297

26. Stott K, Watson M, Howe FS et al (2010) Tail-mediated collapse of HMGB1 is dynamic and occurs via differential binding of the acidic tail to the A and B domains. J Mol Biol 403, 706-722
27. Štros M and Vorlíčková M (1990) Non-histone chromosomal protein $\mathrm{HMG} 1$ reduces the histone $\mathrm{H} 5$-induced changes in c.d. spectra of DNA: the acidic C-terminus of HMG1 is necessary for binding to H5. Int. J Biol Macromol $12,282-288$

28. Cato L, Stott K, Watson M and Thomas JO (2008) The interaction of HMGB1 and linker histones occurs through their acidic and basic tails. J Mol Biol 384, 1262-1272

29. Sutrias-Grau M, Bianchi ME and Bernues J (1999) High mobility group protein 1 interacts specifically with the core domain of human TATA box-binding protein and interferes with transcription factor IIB within the pre-initiation complex. J Biol Chem 274, 1628-1634

30. Das D and Scovell WM (2001) The binding interaction of HMG-1 with the TATA-binding protein/TATA complex. J Biol Chem 276, 32597-32605

31. Ge H and Roeder RG (1994) The high mobility group protein HMG1 can reversibly inhibit class II gene transcription by interaction with the TATA-binding protein. J Biol Chem 269, 17136-17140

32. Bonaldi T, Längst $G$, Strohner R et al (2002) The DNA chaperone HMGB1 facilitates ACF/CHRAC-dependent nucleosome sliding. EMBO J 21, 6865-73

33. Cereghini S and Yaniv M (1984) Assembly of transfected DNA into chromatin: Structural changes in the origin-promoter-enhancer region upon replication. EMBO J 3, 1243-1253

34. Ford JM and Hanawalt PC (1995) Li-Fraumeni syndrome fibroblasts homozygous for p53 mutations are deficient in global DNA repair but exhibit normal transcription-coupled repair and enhanced UV resistance. Proc Natl Acad Sci U S A 92, 8876-8880

35. Hwang BJ, Ford JM, Hanawalt PC and Chu G (1999) Expression of the p48 xeroderma pigmentosum gene is p53-dependent and is involved in global genomic repair. Proc Natl Acad Sci U S A 96, 424-428

36. Fitch ME, Cross IV, Turner SJ et al (2003) The DDB2 nucleotide excision repair gene product p48 enhances global genomic repair in p53 deficient human fibroblasts. DNA Repair 2, 819-826

37. Banerjee S, de Freitas A, Friggeri A, Zmijewski JW, Liu G and Abraham E (2011) Intracellular HMGB1 negatively regulates efferocytosis. J Immunol 187, 4686-4694 